\title{
Correspondence of Plasma and Salivary Cortisol Patterns in Women with Breast Cancer
}

\author{
Jamie M. Zeitzer ${ }^{\mathrm{a}, \mathrm{b}} \quad$ Bita Nouriani $^{\mathrm{a}} \quad$ Eric Neri $^{\mathrm{a}} \quad$ David Spiegel $^{\mathrm{a}}$ \\ ${ }^{a}$ Department of Psychiatry and Behavioral Sciences, Stanford University, Stanford, Calif., and ${ }^{b}$ Mental Illness \\ Research Education and Clinical Center, VA Palo Alto Health Care System, Palo Alto, Calif., USA
}

\author{
Key Words \\ Cortisol · Diurnal rhythm · Saliva · Plasma · Sleep · \\ Breast cancer $\cdot$ Women $\cdot$ Circadian rhythm
}

\begin{abstract}
Introduction: The 'diurnal slope' of salivary cortisol has been used as a measure of stress and circadian function in a variety of reports with several detailing its association with cancer progression. The relationship of this slope, typically a negative value from high morning concentrations to low evening concentrations, to the underlying daily variation in total plasma cortisol throughout the 24-hour cycle, however, has never been reported. Methods: To examine the relationship between the diurnal salivary cortisol slope and the underlying pattern of plasma cortisol in individuals with cancer, we examined a cohort of women with advanced breast cancer ( $n=97$ ) who had saliva and plasma collected during a modified 24-hour, constant posture protocol. Results: We found that the steepness of the diurnal slope of salivary cortisol was correlated with the amplitude of plasma cortisol rhythm when the slope was calculated from samples taken at wake + 30 min and 9 PM $(r=-0.29$, p > 0.05). Other variants of salivary slope calculations were not significantly correlated with the amplitude of the plasma cortisol rhythm. Diurnal salivary cortisol slope steepness was not correlated with the time between habitual waking and the computed circadian peak of cortisol, but there was a correlation between diurnal slope
\end{abstract}

steepness and the time between habitual waking and the time of the awakening spike of morning cortisol ( $r$ values $<-0.23$, p values <0.05). Conclusion: It therefore appears that in women with advanced breast cancer, diurnal salivary cortisol slope primarily represents aspects of the cortisol awakening response in relation to evening levels more than the circadian rhythm of total plasma cortisol.

ㄷ) 2014 S. Karger AG, Basel

\section{Introduction}

Many studies have reported on a 'diurnal slope' of salivary cortisol concentrations during waking hours, with the slope representing the change in salivary cortisol concentrations between the morning (typically at or near wake time) and evening (typically at or near bed time). The normal pattern is described as a negative slope (higher in the morning, lower in the evening), though not all healthy individuals exhibit this pattern [1]. An altered diurnal slope of cortisol has been reported in association with a variety of pathologies, including, but certainly not limited to, breast cancer [2,3], ovarian cancer [4], lung cancer [5], coronary artery disease [6], fibromyalgia [7], depression [8], early-life abuse [9] and disrupted sleep [10]. Flattening of the diurnal slope has been found to predict more rapid disease progression and shorter survival in breast [2], ovarian [4] and lung [5] cancers. Many

\section{KARGER}

E-Mail karger@karger.com

www.karger.com/nen (c) 2014 S. Karger AG, Basel

0028-3835/14/1003-0153\$39.50/0
Jamie M. Zeitzer

3801 Miranda Avenue (151Y)

Palo Alto CA 94304 (USA)

E-Mail jzeitzer@stanford.edu 
of these reports use the diurnal slope of cortisol to impute the strength or normality of circadian rhythms. There is a robust circadian variation in the release of cortisol from the adrenal cortex such that levels begin to rise prior to habitual wake time and reach a nadir in the evening [11]. There is a small, suppressive effect of sleep on plasma cortisol patterns [12], but otherwise, the underlying 24-hour pattern of cortisol is believed to be primarily driven by the suprachiasmatic nuclei, the central circadian pacemaker in mammals [11]. Salivary concentrations of cortisol are well correlated with free (unbound) plasma concentrations of cortisol as this fraction of plasma cortisol moves freely between the blood and salivary compartments [13]. There is, therefore, logical reason to connect the pattern of salivary cortisol with underlying circadian timing.

There are, however, several reasons why the diurnal slope of daytime salivary cortisol might not accurately represent the underlying 24-hour circadian pattern of plasma cortisol. First, as salivary cortisol is often collected in circumstances in which the environment is not controlled (i.e. ambulatory), concentrations may be subject to acute stressors more so than in the laboratory environment in which plasma cortisol is collected. For example, an acute stress in the evening that evoked a spike of cortisol release could result in a positive diurnal slope even though the underlying circadian rhythm was normal. A second issue is that the diurnal slope is dependent on the circadian phase angle of entrainment - the relative position of the circadian rhythm of cortisol to the timing of sleep. For example, if the rhythm of cortisol were phase delayed (later than normal), the morning saliva sample would be obtained prior to the peak of cortisol. Likewise, a phase-advanced rhythm (earlier than normal) would have the morning sample being collected after the peak and the evening sample being collected during the rising phase. Under both of these circumstances, the underlying circadian rhythm of cortisol would be of normal shape and amplitude, but the timing vis-à-vis sleep would be abnormal and the diurnal slope would be flat or positive due to a lower-than-peak morning sample. A third possible issue relates to the pulsatile release of cortisol. Cortisol is released in a highly pulsatile manner. Infrequent sampling, such as occurs with saliva samples, makes determination of a diurnal slope more prone to the vagaries of this pulsatility. For example, if a morning saliva sample is obtained just prior to pulse and an evening sample is obtained at the peak of a pulse, the slope would appear flat despite a normal underlying diurnal rhythm.

To determine whether differences in salivary diurnal slope are related to real or perceived differences in the underlying circadian rhythm, we compared the complete, diurnal pattern of plasma cortisol with that of contemporaneously collected salivary cortisol and the calculated diurnal slope of salivary cortisol concentrations. We did this comparison in a group of women with advanced breast cancer, a population in whom diurnal cortisol has frequently been reported $[2,3,14,15]$.

\section{Materials and Methods}

\section{Participants}

Ninety-seven women diagnosed with advanced breast cancer were recruited to participate. Participants had to meet the following inclusion criteria: documented metastatic or recurrent breast cancer, aged between 45 and 75 years, proficient in English, willing to travel to Stanford, Calif., for 3 nights, postmenopausal, nonsmoking, Karnofsky ratings of $70 \%$ or higher [16], and at least a high school diploma or equivalent. Exclusion criteria were: bilateral lymph node removal, low hematocrit, active cancers (other than breast cancer, basal cell or squamous cell carcinomas of the skin, or in situ cancer of the cervix) within the past 10 years, any concurrent medical condition likely to influence short-term survival, use of corticosteroids, glucocorticoids, benzodiazepines or melatonin within the week preceding and during the in-laboratory study, history of major psychiatric illness that required hospitalization in the preceding year, current substance/alcohol abuse/dependence, or engagement in shift work during 3 months prior to the study. We did allow participants to be on chronic (>1 month), stable doses of antidepressant medications and medications to ameliorate pain; post hoc analyses found that the use of these medications did not affect the results presented below. We examined individual cortisol profiles of women who had been on long-term glucocorticoids or corticosteroids to ensure that the use of such medications did not explain any of the findings described below. Participants were required to abstain from traveling 2 or more time zones away from their local time zone for the 2 weeks prior to and during their participation in the study. If not living within the $\mathrm{Pa}$ cific time zone, participants agreed to maintain their local time zone schedule during a 3-night visit to California.

Participants were recruited through the Stanford Cancer Center, the Army of Women website, and posting of study flyers on the Internet and at various bay area clinics. All procedures were approved by the Stanford University Institutional Review Board functioning according to the third edition of the Guidelines on the Practice of Ethical Committees in Medical Research issued by the Royal College of Physicians of London. Consent was obtained from each participant after full explanation of the purpose and nature of all procedures used.

\section{Protocol}

Using a combination of sleep logs and wrist-worn actigraphs (Actiwatch 2, Philips-Respironics, Bend, Oreg., USA), ambulatory sleep/wake patterns were recorded in all participants for 2 weeks. Actigraphs record 3-dimensional arm movement, which is useful for determining gross patterns of sleep and wake [17]. Actigraph data were interpreted using commercially available software (Actiware 5, Respironics) in combination with the sleep logs. From 
these data, habitual bed and wake times were calculated from the average bed and wake times on the 14 recorded days. Divergences greater than $60 \mathrm{~min}$ from these averages were excluded from the calculations.

Following the 2-week at-home protocol, individuals participated in a 27-hour stay at either the Clinical Translational Research Unit or the Stanford Sleep Disorders Clinic at Stanford Hospital. Rooms were modified to control ambient light exposure and allow for simultaneous recording of sleep and sampling of blood without sleep disruption. Participants arrived at Stanford Hospital approximately $7 \mathrm{~h}$ after habitual wake time (e.g. $3 \mathrm{PM}$ for an individual who habitually woke at $8 \mathrm{AM}$ ). After approximately $1 \mathrm{~h}$ of orientation and medical screening (hematocrit, vital signs), participants entered their room and stayed in this room for the duration of their study, which was scheduled to end approximately $10 \mathrm{~h}$ after habitual wake time the next day (e.g. 6 PM for an individual who habitually woke at $8 \mathrm{AM}$ ). During hours of scheduled wakefulness, participants typically read, did light stretching (strenuous exercise was not allowed) or talked to staff members. An 8-hour period of darkness was scheduled to occur starting $4 \mathrm{~h}$ before the midpoint of habitual sleep and wake times. Lighting during periods of scheduled wake was $\operatorname{dim}(<15 \mathrm{~lx})$, and lights were off $(<0.05 \mathrm{~lx})$ during the period of scheduled sleep. To avoid the metabolic consequences of large meals, participants were provided equicaloric hourly snacks, the sum total of which satisfied their nutritional requirements for the duration of the study [18].

Blood samples were collected via an indwelling venous catheter kept patent with a $0.9 \%$ sodium chloride/1,000 U heparin solution delivered at a $10 \mathrm{ml} / \mathrm{h}$ drip rate. The catheter was placed in the arm contralateral to any axillary lymph node resection. The catheter was attached to the infusion pump with 1.5- or 3.0-meter extension tubing, the former being used during the participant's waking hours and the latter being used during the participant's scheduled sleep time. During scheduled sleep, blood samples were collected in an adjacent room, so as not to disturb the participant's sleep, with the intravenous tubing passing through a shielded porthole in the wall. Blood samples for determination of cortisol concentrations were collected at 20 - to 60-min intervals, with the elevated collection rate occurring from $3 \mathrm{~h}$ prior to habitual bed time until $1 \mathrm{~h}$ after habitual wake time.

\section{Plasma Cortisol Assays and Data Fitting}

At least $1 \mathrm{ml}$ of whole blood was collected via the catheter into a tube coated with ethylenediaminetetraacetic acid. Samples were immediately spun for $15 \mathrm{~min}$ at $1,300 \mathrm{~g}$ in a centrifuge at $4^{\circ} \mathrm{C} ; 40$ $\mu \mathrm{l}$ of the resultant plasma was decanted and stored at $-80^{\circ} \mathrm{C}$ until assay. All samples were assayed in duplicate using a commercially available solid-phase enzyme-linked immunosorbent assay (Immuno-Biological Laboratories, Minneapolis, Minn., USA). Briefly, plasma samples for an individual participant were brought to room temperature, and $20 \mu \mathrm{l}$ of plasma was added into a single well on a 96-well plate. To each sample, $200 \mu \mathrm{l}$ of enzyme conjugate (cortisol conjugated to horseradish peroxidase) was added, mixed and incubated at room temperature for $60 \mathrm{~min}$. The plate was washed (Wellwash AC, Thermo, Waltham, Mass., USA) and dried before $100 \mu \mathrm{l}$ of a substrate solution (tetramethylbenzidine) was added to each well, followed by a 15-min incubation at room temperature. The reaction was stopped with $100 \mu \mathrm{l}$ of stop solution ( $1 \mathrm{~N}$ acid), and the plate was read by a microplate photometer (Multiskan, Fisher Scientific, Pittsburgh, Pa., USA) at $450 \mathrm{~nm}$.
Standards $(0,20,50,100,200,400,800 \mathrm{ng} / \mathrm{ml})$ were processed in a manner identical to the samples. After adjusting for a blank well, results from the standards assay were fit with a 4-parameter logistic model, from which concentrations of the unknowns were calculated. Data quality was ensured with an internal control and reviews of each curve fit and individual sample results. Intra-assay coefficients of variation for 43.5, 226.5 and $403.6 \mathrm{ng} / \mathrm{ml}$ were 8.1, 3.2 and $5.6 \%$, respectively. Interassay coefficients of variation for 55, 209 and $361 \mathrm{ng} / \mathrm{ml}$ were 6.6, 7.7 and 6.5\%, respectively. Assay sensitivity was $2.5 \mathrm{ng} / \mathrm{ml}$.

Plasma cortisol has a characteristic diurnal rhythm, with a nadir occurring around the time of habitual bedtime and a peak occurring just after wake time. This pattern is only slightly altered by the presence of sleep. There are many different curve fitting techniques that have been used to describe the diurnal cortisol rhythm, including cosinor [19], 2-hour smoothing [20], L ${ }^{2}$ norm approximation [21] and harmonic regression [22]. Based on preliminary analyses from an already published data set (data not shown) [23], we found that harmonic regression analysis using a 3-harmonic regression analysis was robust, and for the phase of the first harmonic to be most representative of circadian phase, as indicated by the timing of the rhythm of plasma melatonin [22]. As such, we fit all plasma cortisol data with a 3-harmonic regression analysis (OriginPro 8.0, Microcal, Northampton, Mass., USA), with fixed harmonics (waveforms) of 24, 12 and $8 \mathrm{~h}$. Using this technique, we were able to characterize the time of the peak and trough of the composite curve, the peak of the first harmonic (a marker of circadian phase), the amplitude of the curve (one-half peak-totrough), the mesor (midline estimating statistic of rhythm) and the goodness of fit (adjusted $\mathrm{r}^{2}$ ). We then calculated phase angles between various markers (i.e. the time between two definable portions of rhythmic behavior), including habitual wake time and the peak of the composite curve and habitual wake time and the peak of the first harmonic. The timing of the peak of the first harmonic relative to habitual wake time is a useful marker of the phase angle of entrainment - that is the position of the circadian system relative to the normal timing of sleep.

\section{Salivary Cortisol Assays and Data Fitting}

Saliva samples were collected at 3 time points -9 PM on day 1 , awakening on day 2 and $30 \mathrm{~min}$ after awakening on day 2 . These times were selected as they are commonly used in this area of research [24], although there is considerable variation in the timing of samples used to determine the diurnal slope of salivary cortisol [25]. Three days of saliva sampling are posited as ideal to determine slope [24], so our use of a single day limits some of the statistical reliability of our analyses. As the $9 \mathrm{PM}$ sample was taken on day 1, we carried forward the value of this sample as 9 PM on day 2. Given relatively little drift of the human circadian timing system during this type of protocol [26], we believe this is a valid imputation. Saliva was obtained by having the participant chew on an untreated cotton swab, which was then stored in a plastic container (Salivette, Sarstedt, Newton, N.C., USA). The Salivette was stored at $-80{ }^{\circ} \mathrm{C}$ until assay. For assessment of salivary concentration of cortisol, the Salivette was brought to room temperature and then spun for $15 \mathrm{~min}$ at $1,500 \mathrm{~g}$ in a room temperature centrifuge, with the Salivette filtering out the particulate matter in the saliva. Salivary cortisol concentrations were determined in duplicate with a commercially available enzyme immunoassay kit (Salimetrics, State College, Pa., USA). In brief, $24 \mu \mathrm{l}$ of sample was added to an 
individual well on a 96-well plate. After adding $200 \mu \mathrm{l}$ of conjugate (cortisol conjugated to horseradish peroxidase) to each well, the plate was rotated for $5 \mathrm{~min}$ at $500 \mathrm{rpm}$ and then left at room temperature for $55 \mathrm{~min}$. The plate was washed and dried before $200 \mu \mathrm{l}$ of a tetramethylbenzidine solution was added to each well. The plate was again rotated and left in the dark at room temperature for $25 \mathrm{~min}$. The reaction was stopped with $50 \mu \mathrm{l}$ of a stop solution, the plate was rotated for $3 \mathrm{~min}$, and then the plate was read by the microplate photometer at $450 \mathrm{~nm}$. Standards (0, 0.012, 0.037, $0.111,0.333,1.000,3.000 \mu \mathrm{g} / \mathrm{dl})$ were processed in a manner identical to the subject samples. After adjusting for a blank well, results from the standards assay were fit with a 4-parameter logistic model, from which concentrations of the unknowns were calculated. Data quality was ensured with commercially available controls and reviews of each curve fit and individual sample results. Intra-assay coefficients of variation for 0.097 and $0.999 \mu \mathrm{g} / \mathrm{dl}$ were 3.65 and $3.35 \%$, respectively. Interassay coefficients of variation for 0.101 and $1.020 \mu \mathrm{g} / \mathrm{dl}$ were 6.41 and $3.75 \%$, respectively. Assay sensitivity was $0.003 \mu \mathrm{g} / \mathrm{dl}$.

\section{Statistics}

Summary data were generated using Excel (v.11.8332.8333, Microsoft, Redmond, Wash., USA). Regression analyses were completed using OriginPro (Origin Labs, Northampton, Mass., USA). Functional principal component analyses (fPCA) were completed using R [27, 28]. fPCA is similar to PCA except that it was applied to semicontinuous data (plasma cortisol) rather than discrete data. In this PPCA, participants who had cortisol concentrations available from at least 38 of 51 plasma samples and no greater than a gap of $1 \mathrm{~h}$ of missing data were used $(\mathrm{n}=61)$. Included data gaps (3.3\% of sample) were filled by linear interpolation such that all participants had a continuous string of $24 \mathrm{~h}$ of data with a data point every $20 \mathrm{~min}$. Data from individual participants were fit with a 9-Fourier-based function. These equations were then subjected to functional data analysis that computed the equations underlying the variance in the Fourier equations [29]. The relative weight of each of these underlying equations (fPCA component) was calculated for each participant and represented as an eigenvalue that could be subjected to parametric statistics. The first fPCA component explains the greatest amount of variance and each subsequent component explains less. We calculated the first 4 components of the fPCA as these typically explain most of the variance and remaining components often have low probative value ( $<1 \%$ variance explained). Each eigenvalue represents the relative amount that a cortisol pattern is represented by a specific equation or shape. For example, the first fPCA component represented the amplitude differences between subjects without changes to the overall shape of the cortisol pattern. The first 4 components of the fPCA were compared with diurnal slope of salivary cortisol concentrations.

Data are presented as means \pm SD.

\section{Results}

\section{General Results}

Of the 97 women with advanced breast cancer who took part in the protocol, saliva samples were available from 91 (age: $57.4 \pm 7.58$ years, body mass index $=28.1 \pm$ 5.78). Salivary concentrations of cortisol were $0.33 \pm 0.19$ $\mu \mathrm{g} / \mathrm{dl}$ at wake time $(\mathrm{n}=91), 0.38 \pm 0.20 \mu \mathrm{g} / \mathrm{dl}$ at wake + $30 \min (n=86)$ and $0.077 \pm 0.12 \mu \mathrm{g} / \mathrm{dl}$ at $9 \mathrm{PM}(\mathrm{n}=91)$. In 83 of the women (age: $57.7 \pm 7.80$ years, body mass in$\operatorname{dex}=28.0 \pm 5.76$ ), there were contemporaneous blood samples obtained at these 3 times. Plasma concentrations of cortisol were $143.0 \pm 49.90 \mathrm{ng} / \mathrm{ml}$ at wake time $(\mathrm{n}=77)$, $154.7 \pm 46.30 \mathrm{ng} / \mathrm{ml}$ at wake $+30 \mathrm{~min}(\mathrm{n}=72)$ and $30.46 \pm$ $23.98 \mathrm{ng} / \mathrm{ml}$ at $9 \mathrm{PM}(\mathrm{n}=82)$. For both the saliva and plasma, due to single missing samples in individual participants, the totals at each time point vary. Each of the pairs of blood and saliva samples was obtained within 30 min of one another $(6.41 \pm 4.78 \mathrm{~min})$. Plasma and salivary concentrations were well correlated with one another ( $\mathrm{n}=229$, adjusted $\mathrm{r}^{2}=0.47, \mathrm{p}<0.001$, linear regression), with salivary concentrations of cortisol being $2.6 \pm 2.3 \%$ of those which are found in plasma. Thus, as anticipated, the cortisol concentration within each individual salivary sample was reliably indicative of the plasma concentration of cortisol.

\section{Salivary Slope versus Plasma Curve Fitting}

Given the analytic variability in the literature [25], we calculated the diurnal slope of salivary cortisol in a variety of ways, each using regression analysis: (1) wake sample to 9 PM sample, (2) wake sample to 9 PM sample with both samples natural log transformed prior to calculation of slope [24], (3) wake time +30 min sample to 9 PM sample, and (4) wake + 30 min sample to 9 PM sample with both samples natural log transformed prior to calculation of slope. The diurnal slope was $-0.018 \pm 0.016$ $(\operatorname{method} 1, \mathrm{n}=91),-0.12 \pm 0.084(\operatorname{method} 2, \mathrm{n}=91)$, $-0.023 \pm 0.015(\operatorname{method} 3, \mathrm{n}=90)$, and $-0.13 \pm 0.095$ (method 4, $\mathrm{n}=90$ ). Methods 1 and 3 (untransformed data) were moderately correlated $(\mathrm{r}=0.39, \mathrm{p}<0.001)$ while methods 2 and 4 (natural log-transformed data) were quite strongly correlated $(\mathrm{r}=0.86, \mathrm{p}<0.001)$. This is expected as the natural log transformation decreases the impact of elevated values, such as occurs at the wake + 30 min sample, yielding relatively little difference in the slope calculated from such transformed data when the data are anchored to either the wake sample or the wake + 30 min sample.

To examine our a priori hypothesis that the diurnal slope of salivary cortisol was related to the position (i.e. phase angle) of the circadian system relative to the timing of sleep, we ran regression analyses between circadian phase angle (time between habitual wake time and the peak of the first harmonic of the 3-harmonic fit to the 
Table 1. Simple linear regression (r) of 4 different methods of diurnal slope calculation against 4 measures derived from 3-harmonic regression analysis of $24 \mathrm{~h}$ of plasma cortisol data

\begin{tabular}{|c|c|c|c|c|c|}
\hline \multicolumn{2}{|c|}{$\begin{array}{l}\text { Method of calculation diurnal } \\
\text { slope of salivary cortisol }\end{array}$} & \multirow{2}{*}{$\begin{array}{l}\Psi(\mathrm{HWT}-\mathrm{P} 3), \mathrm{h} \\
-0.25^{*}\end{array}$} & \multirow{2}{*}{$\begin{array}{l}\Psi \text { (HWT-P1), h } \\
0.07\end{array}$} & \multirow{2}{*}{$\begin{array}{l}\begin{array}{l}\text { Amplitude, } \\
\mu \mathrm{g} / \mathrm{dl}\end{array} \\
-0.10\end{array}$} & \multirow{2}{*}{$\begin{array}{l}\begin{array}{l}\text { Mesor, } \\
\mu \mathrm{g} / \mathrm{dl}\end{array} \\
0.08\end{array}$} \\
\hline (1) & Wake $\rightarrow 9 \mathrm{PM}$ & & & & \\
\hline (2) & $\ln ($ wake $) \rightarrow \ln (9 \mathrm{PM})$ & $-0.23^{*}$ & 0.09 & -0.04 & 0.14 \\
\hline (3) & Wake $+30 \rightarrow 9 \mathrm{PM}$ & -0.19 & -0.07 & $-0.29 *$ & -0.06 \\
\hline (4) & $\ln ($ wake +30$) \rightarrow \ln (9 \mathrm{PM})$ & $-0.23^{*}$ & -0.05 & -0.11 & 0.08 \\
\hline
\end{tabular}

The 4 salivary slopes (all 2-point linear regression): wake time and 9 PM concentrations, natural log of wake time and 9 PM concentrations, wake time +30 min and 9 PM concentrations, and natural log of wake time +30 min and 9 PM concentrations. The 4 variables derived from plasma cortisol: the phase angle $(\psi)$ between habitual wake time (HWT) and the peak of the composite 3-harmonic model (P3), the phase angle between habitual wake time and the peak of the first harmonic (P1), the amplitude (one-half peak-to-trough) of the fit, and the mesor of the fit. ${ }^{*} \mathrm{p}<0.05$, linear regression.

plasma cortisol data) and the diurnal slope (as calculated by 4 methods; available from 78 participants due to missing plasma samples). We could, however, find no such association between diurnal slope and the phase angle of the circadian timing of cortisol (table 1).

To examine our other a priori hypothesis that the diurnal slope of salivary cortisol was related to the amplitude of the plasma cortisol rhythm, we ran simple regression analyses between the amplitude of the 3-harmonic fit to the plasma cortisol data and the salivary diurnal slope (as calculated by 4 methods). Diurnal amplitude of the 3-harmonic fit to the plasma cortisol was significantly correlated with the salivary diurnal slope only when the slope was calculated using the untransformed wake $+30 \mathrm{~min}$ as the morning value (table 1), which would be the slope calculated from the presumptive highest to lowest points in the diurnal cortisol curve. Given that the log transformation is used to remove variability associated with peak cortisol values, the lack of association with log-transformed slopes is not altogether unsurprising. We also examined whether the mesor of the 3-harmonic fit to the plasma cortisol data was associated with the salivary diurnal slope, but could find no such relationship (table 1).

The wake $+30 \mathrm{~min}$ time point is likely to represent the cortisol awakening response - an extra elevation in the already high early morning concentrations that usually follows a morning awakening. As it was the slope of the untransformed data using the wake +30 min time point of saliva collection that was associated with the amplitude of plasma cortisol, we also examined whether the phase angle of habitual wake time with the peak of the composite curve of the 3-harmonic fit (presumably influenced more by the awakening response than the peak of the first

Salivary and Plasma Cortisol in Breast Cancer harmonic of the 3-harmonic fit) had a relationship to the diurnal slope of salivary cortisol. The diurnal slopes as calculated using either the log-transformed data (both techniques) and the nontransformed data (wake to 9 PM only) were indeed associated with the phase angle between habitual wake time and the peak of the 3-harmonic fit (table 1). Thus, the greater the temporal distance between the peak of the cortisol rhythm and habitual wake time, the less steep the slope. Thus, as a group, a 'flattened' diurnal rhythm in salivary cortisol is representative of a diminution of both the amplitude of the overall plasma cortisol rhythm and a cortisol peak that is abnormally timed visà-vis the timing of sleep. Note, the timing of the circadian system as indicated by the time of the peak of the first harmonic is not associated with this flattening - rather it is a delay in the morning awakening response of cortisol that is associated with the flattening of the diurnal slope.

\section{Salivary Slope versus Plasma fPCA}

Of the 91 women in whom we were able to calculate the diurnal slope of salivary cortisol, $10(11 \%)$ had a positive slope (fig. 1). As can be seen in the figure (note, due to missing blood, there are only 8 of the 10 individuals in fig. 1), there appear to be a variety of 'reasons' underlying the positive value of the salivary slope. In order to analyze these patterns and determine if a specific pattern of plasma cortisol was associated with a positive or flatter diurnal slope of salivary cortisol, the plasma cortisol data were subjected to fPCA and then compared with salivary diurnal slope (described in the Methods). Complete plasma data were available from 61 women, 6 of whom (10\%) had a positive diurnal slope of salivary cortisol. The first 4 components of the fPCA were able to explain $81.8 \%$ of the variance of the plas- 


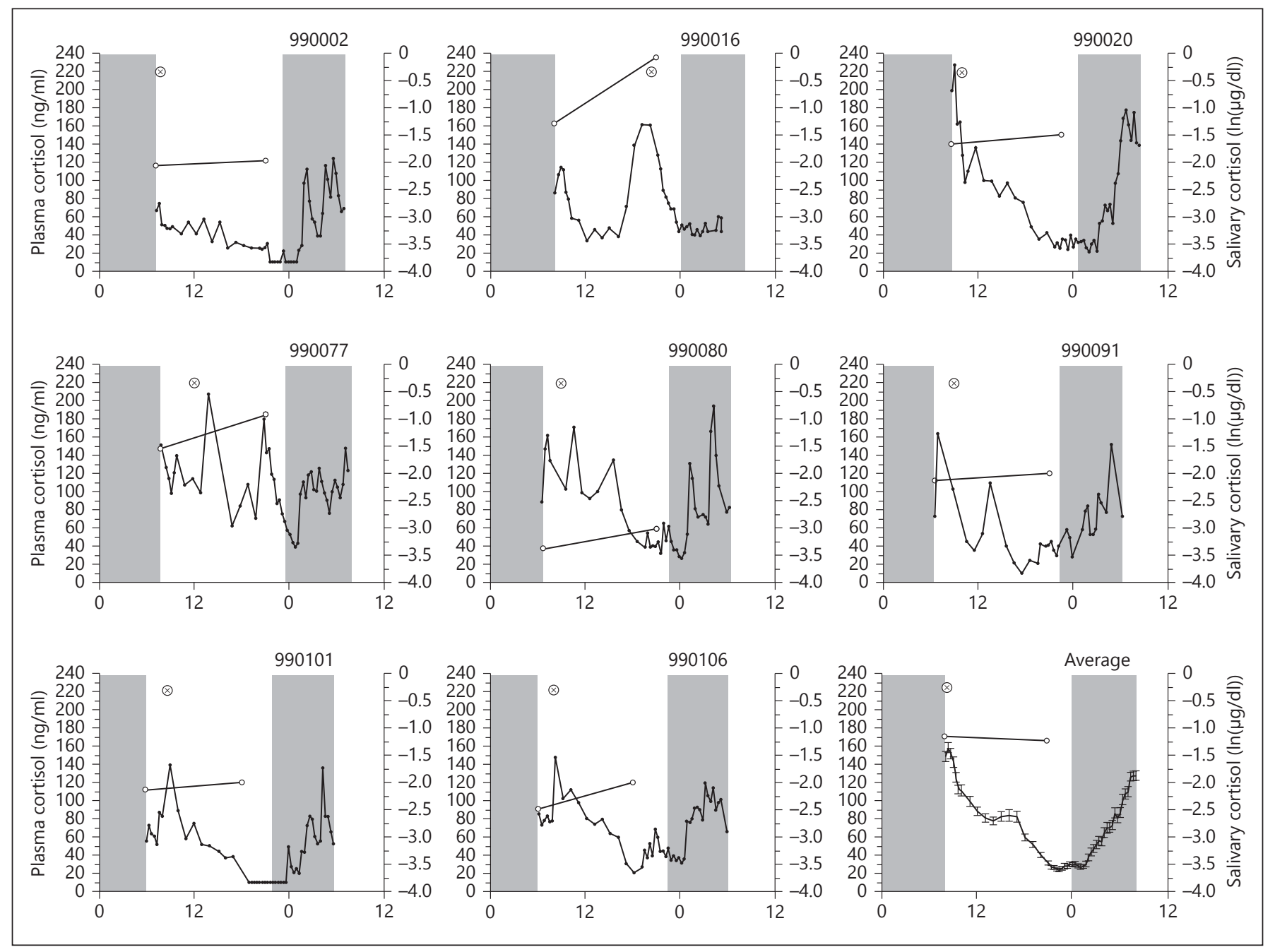

Fig. 1. Individual plots of participants with positive diurnal salivary cortisol slopes. Each panel (except the lower right) has data collected during the protocol for the 8 participants with positive slopes. The average \pm SEM curves for the remaining available participants $(\mathrm{n}=75)$ is shown in the lower right panel. The cortisol concentra-

ma cortisol data. In comparing the first 4 components to the diurnal slope, the first (approx. $\mathrm{r}^{2}=-0.02, \mathrm{p}=0.90$; linear regression), second (approx. $\mathrm{r}^{2}=0.02, \mathrm{p}=0.17$; linear regression) and fourth (approx. $\mathrm{r}^{2}=0.01, \mathrm{p}=0.19$; linear regression) components of the PCA did not appear related to the diurnal slope. The third component of the fPCA, however, was linearly related to the diurnal slope of salivary cortisol (approx. $\mathrm{r}^{2}=0.08, \mathrm{p}<0.05$; linear regression). Examination of both the individual plots (fig. 1) and the results of the fPCA (fig. 2) reveal that in some participants, the flattening of the slope is associated with a lower morning peak and an elevated evening nadir (red and blue lines in fig. 2). tion for each plasma sample (left y-axis, solid circles) and saliva sample (right y-axis, open circles) is plotted at the precise time of collection (wake and $9 \mathrm{PM}$ ). The gray boxes represent the timing of lightsoff when sleep was permitted. The circadian phase $(\otimes$, peak of the first harmonic of the 3-harmonic fit) is plotted for each participant.

\section{Discussion}

Our data indicate that there is no single, underlying plasma pattern that is represented by the observation of a flat or positive diurnal slope of salivary cortisol. Rather, from a statistical standpoint, there appear to be 2 main associations between diurnal slope and the plasma pattern of cortisol. First, in some individuals, a delay in the fitted peak of the 3-harmonic curve, but not the circadian peak of plasma cortisol, is associated with a flattening of the salivary diurnal slope. This may be a slightly later than normal peak (e.g. participant No. 990016) or a peak with 


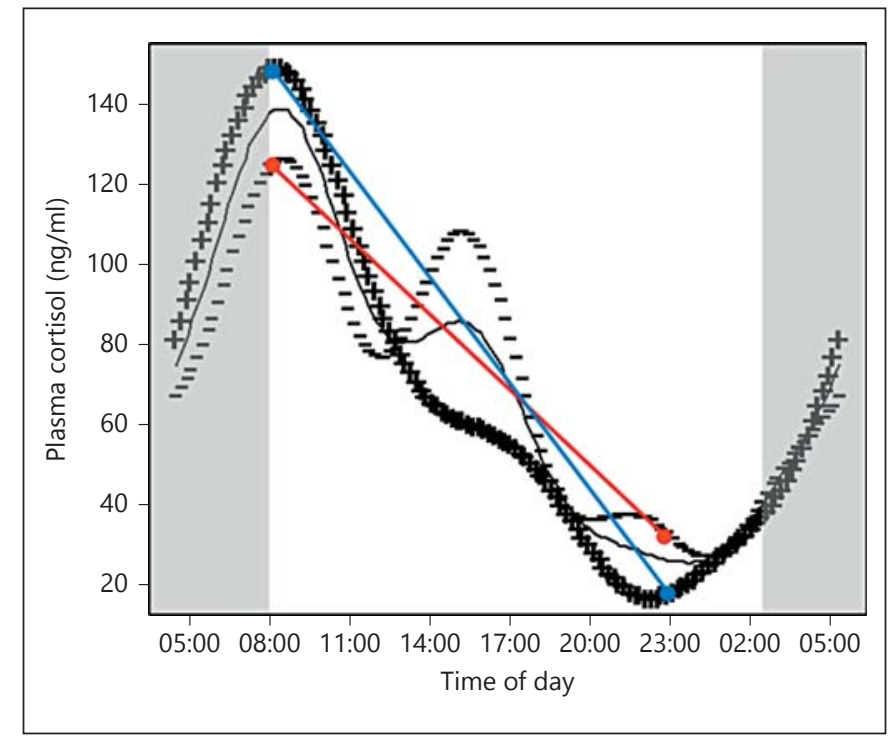

Fig. 2. fPCA component 3 and its relationship to diurnal salivary cortisol slope. Data from high $(+++)$ and low (--) eigenvalues of fPCA component 3 are plotted along with the average (solid gray line) cortisol curve of all participants. The gray boxes represent the relative time of sleep. The red and blue lines represent the possible salivary slopes as might be derived from a sample taken at wake time and 9 PM, from individuals with higher (blue) or lower (red) fPCA component 3 scores.

highly aberrant timing (e.g. participant No. 990101). The flat slope would, therefore, be primarily due to the morning saliva sample being obtained before the actual peak in morning cortisol. As there was no association between the steepness of the slope and the relative timing of the circadian clock (phase angle between the time of the first harmonic and the timing of sleep), this finding is unlikely to represent an abnormality of circadian timing. Rather, it is more likely to represent a delay in the so-called cortisol awakening response [30-32]. Confirmation of circadian timing with an independent marker such as melatonin, however, would be necessary to confirm the normality of circadian timing.

A second association is with a pattern that has a slightly reduced morning peak and an elevated nadir in plasma cortisol in the evening (e.g. participant No. 990080). The flat slope would be, therefore, due to the morning sample being slightly lower than normal and the evening sample being slightly higher than normal. This pattern has been observed before in individuals with insomnia [33] and in older individuals [34]. It is not likely due to chronic stress or depression, both of which are likely to alter the constitutive expression of cortisol, as these are sometimes characterized by constantly low (chronic stress) or elevated (depression) levels of plasma cortisol $[35,36]$. As the slope obtained between untransformed salivary cortisol data obtained near the cortisol awakening and the evening was the only diurnal salivary cortisol slope method that was correlated with the diurnal amplitude of plasma cortisol (table 1), use of this slope derivation would appear to be important to accurately capture the underlying amplitude in plasma cortisol concentrations. Use of a slope derived from natural log-transformed data would, however, appear to capture some of the variance associated with the timing of the morning peak of cortisol vis-à-vis its timing relative to habitual wake time.

There are still participants (e.g.participant No. 990020) in whom there is no apparent explanation for a flattened slope. It is possible that there may be an alteration in corticosteroid-binding globulins (transcortin) such that the relationship between total cortisol (plasma) and fractional free cortisol (saliva) could be inconsistent during a single day, possibly due to acute stress [37] or the breast cancer or its treatment [38], including the use of specific drugs that might change the properties of steroid-binding proteins [39]. Further study of free plasma cortisol concentrations versus salivary cortisol concentrations would be necessary to explore this hypothesis. In one study of 109 relatively healthy individuals, $51 \%$ had normal diurnal slopes of salivary cortisol on 2 consecutive days and $17 \%$ had flattened slopes on 2 consecutive days, with the remaining $31 \%$ having a normal slope one day and a flattened slope the other, indicating either a relative lack of reproducibility of a flat slope or a very high interday variability in salivary cortisol slope [40]. To our knowledge, this type of day-to-day variability in diurnal rhythmicity has never been reported for plasma cortisol concentrations in any normal or pathological state, raising the question as to the physiological significance of this 'flat' pattern.

Our data indicate that it is likely that individuals with a flattened diurnal slope of salivary cortisol represent a heterogeneous group. In several, but not all [3], previous studies, including those on women with breast cancer, diurnal slope was not different between controls and patients. The presence of flatter diurnal slopes of salivary cortisol among patients is however associated with reduced survival or other negative health outcomes, implying an important physiological meaning for the construct $[2,4,5,41,42]$. In previous work, flattening of diurnal slopes of salivary cortisol among women with metastatic breast cancer has been shown to be related to a higher 
waking rise of cortisol and reduced adrenal suppression the day after administration of $0.5 \mathrm{mg}$ of dexamethasone, suggesting reduced feedback inhibition once cortisol levels are elevated, rather than hypersensitivity to activation [43].

Our current data indicate that flatter diurnal slopes of salivary cortisol are unlikely to represent an abnormality of the circadian system, but are more likely associated with a change in the morning cortisol awakening response and the timing of salivary sampling in relation to that response. As such, it beckons the question as to the physiological upstream cause of the flattened diurnal slopes that might ultimately be associated with reduced survival and negative health outcomes and why such 'flattening' does not appear to be associated with acute nega- tive outcomes in healthy individuals. Future research on the causal mechanisms of flattened diurnal slopes will be necessary to clarify this physiology.

\section{Acknowledgments}

We thank our research participants for their time, wisdom and willingness to participate, and the Dr. Susan Love Research Foundation's Love/Avon Army of Women Program for their assistance in recruitment. We thank Dr. Dirk Hellhammer for helpful comments on this paper. We also wish to thank the nursing staff of the Clinical Translational Research Unit for staffing the studies and Ms. Chung-Ping Liao and Mr. Ryan Fisicaro for conducting the cortisol assays. This work was supported by the National Cancer Institute (R01CA118567) and the National Center for Research Resources (UL1 RR025744).

\section{References}

1 Stone AA, Schwartz JE, Smyth J, Kirschbaum C, Cohen S, Hellhammer D, Grossman S: Individual differences in the diurnal cycle of salivary free cortisol: a replication of flattened cycles for some individuals. Psychoneuroendocrinology 2001;26:295-306.

-2 Sephton SE, Sapolsky RM, Kraemer HC, Spiegel D: Diurnal cortisol rhythm as a predictor of breast cancer survival. J Natl Cancer Inst 2000;92:994-1000.

3 Abercrombie HC, Giese-Davis J, Sephton S, Epel ES, Turner-Cobb JM, Spiegel D: Flattened cortisol rhythms in metastatic breast cancer patients. Psychoneuroendocrinology 2004;29:1082-1092.

-4 Schrepf A, Clevenger L, Christensen D, Degeest K, Bender D, Ahmed A, Goodheart MJ, Dahmoush L, Penedo F, Lucci JA III, GanjeiAzar P, Mendez L, Markon K, Lubaroff DM, Thaker PH, Slavich GM, Sood AK, Lutgendorf SK: Cortisol and inflammatory processes in ovarian cancer patients following primary treatment: relationships with depression, fatigue, and disability. Brain Behav Immun 2013;30(suppl):S126-S134.

-5 Sephton SE, Lush E, Dedert EA, Floyd AR, Rebholz WN, Dhabhar FS, Spiegel D, Salmon P: Diurnal cortisol rhythm as a predictor of lung cancer survival. Brain Behav Immun 2013;30(suppl):S163-S170.

6 Cohen S, Schwartz JE, Epel E, Kirschbaum C, Sidney S, Seeman T: Socioeconomic status, race, and diurnal cortisol decline in the Coronary Artery Risk Development in Young Adults (CARDIA) Study. Psychosom Med 2006;68:41-50.

7 Weissbecker I, Floyd A, Dedert E, Salmon P, Sephton S: Childhood trauma and diurnal cortisol disruption in fibromyalgia syndrome. Psychoneuroendocrinology 2006;31: 312-324.
8 Stetler C, Dickerson SS, Miller GE: Uncoupling of social zeitgebers and diurnal cortisol secretion in clinical depression. Psychoneuroendocrinology 2004;29:1250-1259.

\9 Van der Vegt EJ, van der Ende J, Kirschbaum C, Verhulst FC, Tiemeier H: Early neglect and abuse predict diurnal cortisol patterns in adults. A study of international adoptees. Psychoneuroendocrinology 2009;34:660-669.

10 Lasikiewicz N, Hendrickx H, Talbot D, Dye L: Exploration of basal diurnal salivary cortisol profiles in middle-aged adults: associations with sleep quality and metabolic parameters. Psychoneuroendocrinology 2008;33: 143-151.

11 Czeisler CA, Klerman EB: Circadian and sleep-dependent regulation of hormone release in humans. Rec Prog Hormone Res 1999;54:97-132.

12 Weitzman ED, Zimmerman JC, Czeisler CA, Ronda J: Cortisol secretion is inhibited during sleep in normal man. J Clin Endocrinol Metab 1983;56:352-358

13 Kirschbaum C, Hellhammer DH: Salivary cortisol; in Fink G (ed): Encyclopedia of Stress. San Diego, Academic Press, 2000, vol 3, pp 379-383.

14 Bower JE, Ganz PA, Dickerson SS, Petersen L, Aziz N, Fahey JL: Diurnal cortisol rhythm and fatigue in breast cancer survivors. Psychoneuroendocrinology 2005;30:92-100.

15 Vedhara K, Tuinstra J, Miles JN, Sanderman R, Ranchor AV: Psychosocial factors associated with indices of cortisol production in women with breast cancer and controls. Psychoneuroendocrinology 2006;31:299-311.

16 Karnofsky DA, Burchenal JH: The clinical evaluation of chemotherapeutic agents in cancer; in MacLeod CM (ed): Evaluation of Chemotherapeutic Agents. New York, Columbia University Press, 1949, pp 191-205.
17 Ancoli-Israel S, Cole R, Alessi C, Chambers M, Moorcroft W, Pollack CP: The role of actigraphy in the study of sleep and circadian rhythms. Sleep 2003;26:342-392.

18 Mifflin MD, St Jeor ST, Hill LA, Scott BJ, Daugherty SA, Koh YO: A new predictive equation for resting energy expenditure in healthy individuals. Am J Clin Nutr 1990;51: 241-247.

19 Halberg F, Johnson EA, Nelson W, Runge W, Sothern RB: Autorhythmometry - procedures for physiologic self-measurements and their analysis. Physiol Teacher 1972;7: $1-11$.

20 Dijk DJ, Duffy JF, Silva EJ, Shanahan TL, Boivin DB, Czeisler CA: Amplitude reduction and phase shifts of melatonin, cortisol and other circadian rhythms after a gradual advance of sleep and light exposure in humans. PLoS One 2012;7:e30037.

21 Chakraborty A, Krzyzanski W, Jusko WJ: Mathematical modeling of circadian cortisol concentrations using indirect response models: comparison of several methods. J Pharmacokinetics Biopharm 1999;27:23-43.

22 Klerman EB, Gershengorn HB, Duffy JF, Kronauer RE: Comparisons of the variability of three markers of the human circadian pacemaker. J Biol Rhythms 2002;17:181193.

23 Zeitzer JM, Dijk D-J, Kronauer RE, Brown EN, Czeisler CA: Sensitivity of the human circadian pacemaker to nocturnal light: melatonin phase resetting and suppression. J Physiol 2000;526:695-702.

24 Kraemer HC, Giese-Davis J, Yutsis M, O’Hara R, Neri E, Gallagher-Thompson D, Taylor $\mathrm{CB}$, Spiegel D: Design decisions to optimize reliability of daytime cortisol slopes in an older population. Am J Geriatr Psychiatry 2006; 14:325-333. 
-25 Adam EK, Kumari M: Assessing salivary cortisol in large-scale, epidemiological research. Psychoneuroendocrinology 2009;34: 1423-1436.

26 Duffy JF, Dijk D-J: Getting through to circadian oscillators: why use constant routines? J Biol Rhythms 2002;17:4-13.

27 R Development Core Team: R: A Language and Environment for Statistical Computing. Vienna, R Foundation for Statistical Computing, 2010.

28 Zeitzer JM, David R, Friedman L, Mulin E, Garcia R, Wang J, Yesavage JA, Robert PH, Shannon W: Phenotyping apathy in individuals with Alzheimer disease using functional principal component analysis. Am J Geriatr Psychiatry 2013;21:391-397.

29 Ramsay JO, Silverman BW: Functional Data Analysis, ed 2. New York, Springer, 2005.

$>30$ Pruessner JC, Wolf OT, Hellhammer DH, Buske-Kirschbaum A, von Auer K, Jobst S, Kaspers F, Kirschbaum C: Free cortisol levels after awakening: a reliable biological marker for the assessment of adrenocortical activity. Life Sci 1997;61:2539-2549.

-31 Pruessner JC, Hellhammer DH, Kirschbaum C: Burnout, perceived stress, and cortisol responses to awakening. Psychosom Med 1999; 61:197-204.

-32 Wust S, Federenko I, Hellhammer DH, Kirschbaum C: Genetic factors, perceived chronic stress, and the free cortisol response to awakening. Psychoneuroendocrinology 2000;25:707-720.
33 Vgontzas AN, Bixler EO, Lin HM, Prolo P, Mastorakos G, Vela-Bueno A, Kales A, Chrousos GP: Chronic insomnia is associated with nyctohemeral activation of the hypothalamic-pituitary-adrenal axis: clinical implications. J Clin Endocrinol Metab 2001;86:37873794.

34 Vgontzas AN, Zoumakis M, Bixler EO, Lin HM, Prolo P, Vela-Bueno A, Kales A, Chrousos GP: Impaired nighttime sleep in healthy old versus young adults is associated with elevated plasma interleukin-6 and cortisol levels: physiologic and therapeutic implications. J Clin Endocrinol Metab 2003;88:2087-2095.

35 Yehuda R, Teicher MH, Trestman RL, Levengood RA, Siever LJ: Cortisol regulation in posttraumatic stress disorder and major depression: a chronobiological analysis. Biol Psychiatry 1996;40:79-88.

36 Linkowski P, Mendlewicz J, Leclercq R, Brasseur M, Hubain P, Golstein J, Copinschi G, Van Cauter E: The 24-hour profile of adrenocorticotropin and cortisol in major depressive illness. J Clin Endocrinol Metab 1985;61:429438.

37 Murphy BEP: Corticosteroid-binding globulin (transcortin); in Fink G (ed): Stress Science: Neuroendocrinology. San Francisco, Elsevier, 2010, pp 245-252.
8 Bell E, Bulbrook RD, Deshpande N: Transcortin in the plasma of patients with breast cancer. Lancet 1967;290:395-397.

39 Löfgren L, Wallberg B, Wilking N, Fornander T, Rutqvist LE, Carlstrom K, von Schoultz B, von Schoultz E: Tamoxifen and megestrol acetate for postmenopausal breast cancer: diverging effects on liver proteins, androgens, and glucocorticoids. Med Oncol 2004;21: 309-318.

40 Smyth JM, Ockenfels MC, Gorin AA, Catley D, Porter LS, Kirschbaum C, Hellhammer $\mathrm{DH}$, Stone AA: Individual differences in the diurnal cycle of cortisol. Psychoneuroendocrinology 1997;22:89-105.

41 Weinrib AZ, Sephton S, Degeest K, Penedo F, Bender D, Zimmerman B, Kirschbaum C, Sood AK, Lubaroff DM, Lutgendorf SK: Diurnal cortisol dysregulation, functional disability, and depression in women with ovarian cancer. Cancer 2010;116:4410-4419.

42 Cohen L, Cole SW, Sood AK, Prinsloo S, Kirschbaum C, Arevalo JMG, Jennings NB, Scott S, Vence L, Wei Q, Kentor D, Radvanyi L, Tannir N, Jonasch E, Tamboli P, Pisters L: Depressive symptoms and cortisol rhythmicity predict survival in patients with renal cell carcinoma: role of inflammatory signaling. PLoS One 2012; 7:e42324.

43 Spiegel D, Giese-Davis J, Taylor CB, Kraemer $\mathrm{H}$ : Stress sensitivity in metastatic breast cancer: analysis of hypothalamic-pituitary-adrenal axis function. Psychoneuroendocrinology 2006;31:1231-1244. 